\title{
Stable Suspensions of Lignocellulose Nanofibrils (LCNFs) Dispersed in Organic Solvents
}

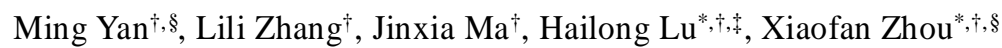 \\ † Jiangsu Co-Innovation Center of Efficient Processing and Utilization of Forest Resources, Jiangsu \\ Provincial Key Lab of Pulp and Paper Science and Technology, College of Light Industry and Food, \\ Nanjing Forestry University, Nanjing 210037, China \\ ‡ Institute of Chemical Industry of Forest Products, Chinese Academy of Forestry, National \\ Engineering Lab for Biomass Chemical Utilization, Key and Open Lab of Forest Chemical \\ Engineering of State Administration of Forestry and Grassland, Key Lab of Biomass Energy and \\ Material of Jiangsu Province. No. 16 Suojinwucun Road, Xuanwu District, Nanjing 210042, China \\ § National-Provincial Joint Engineering Research Center of Electromechanical Product Packaging, \\ Nanjing Forestry University, Nanjing 210037, China
}

*Email: hailonglull@126.com.

*Email: zxiaofan@njfu.com.cn.

The number of pages: 3 pages.

The number of figures: 0 figure.

The number of tables: 0 table. 


\section{Experimental}

Characterization of Samples. The chemical compositions of WS, CLC, and LCNF samples were detected in a two-step sequential acid hydrolysis according to the previous report: (1) 0.3 g of oven-dry sample was weighed to centrifuge tubes and then $3 \mathrm{~mL}$ of $\mathrm{H}_{2} \mathrm{SO}_{4}(72 \% \mathrm{w} / \mathrm{w})$ was added to the centrifuge tubes, mixed well and kept in a water bath at $30{ }^{\circ} \mathrm{C}$ for $60 \mathrm{~min}$; (2) The sample was transferred into the screw cap bottle and then the concentration of $\mathrm{H}_{2} \mathrm{SO}_{4}$ was diluted to $4 \%$ with distilled water. The screw cap bottle was placed in an autoclave for $60 \mathrm{~min}$ at $121^{\circ} \mathrm{C}$ and cooled to the ambient temperature. Then, the obtained sample was filtered by G3 funnel; (3) The residue was washed to neutral with hot distilled water and dried at $105^{\circ} \mathrm{C}$. The oven-dry sample and G3 were put into a laboratory muffle furnace for 5 $\mathrm{h}$ at $550{ }^{\circ} \mathrm{C}$ and then cooled to the ambient temperature. The acid-insoluble lignin (AIL) and ash were calculated gravimetrically and the acid-soluble lignin (ASL) was determined by UV spectroscopy; (4) 5 $\mathrm{mL}$ of filtrate was taken and the $\mathrm{pH}$ was adjusted to 2 with $\mathrm{NaOH}(8 \% \mathrm{w} / \mathrm{w})$. Then, the sample was diluted to $10 \mathrm{~mL}$ and the sugar content of the sample was detected using high-performance liquid chromatography (HPLC). Three parallel samples were used for each test.

Dispersion of the LCNFs. $E_{N R}$ and Kamlet-Taft parameters have been reported as an indicator to characterize the solvatochromic of various conventional solvents, which have been described in previous literature $^{2-7}$, containing molecular solvents, ionic solvents (ILs) and deep eutectic solvents (DESs) and so on. The dyes of NR, NEt2, and $\mathrm{NH}_{2}$ were used to calculate $E_{N R}$ and Kamlet-Taft parameters. Firstly, the dyes were respectively dissolved in dehydrated dichloromethane at a concentration of $0.0004 \mathrm{M}$ and stored at $4^{\circ} \mathrm{C}$. Then $0.2 \mathrm{~mL}$ prepared indicator solution was transferred to the centrifuge tube and evaporated at $50^{\circ} \mathrm{C}$ to remove the dichloromethane.0.8 $\mathrm{mL}$ Ethylene glycol, 1,2-Propanediol, and 1,4Butanediol was respectively add into the centrifuge tube and the final concentration was $0.0001 \mathrm{M}$. UV- 
vis spectral values were measured using Gemini XPS microplate reader (SpectraMax i3x, Molecular Device, UK) to detect the solvatochromic shift of different indicator dyes. The $E_{N R}, \pi^{*}, \alpha$, and $\beta$ values have been calculated by the following equations:3,7

$$
\begin{gathered}
E_{N R}=28591 / \lambda_{\max , N R} \\
\pi^{*}=\frac{\lambda_{\max , \mathrm{NE}}-27.52}{-3.182} \\
\alpha=\frac{19.9657-1.0241 \pi-\lambda_{\max , \mathrm{NR}}}{1.6078} \\
\beta=\frac{\lambda_{\max , \mathrm{NEt}_{2}-\lambda_{\max , \mathrm{NH}_{2}}+2.64}^{2.8}}{}
\end{gathered}
$$

\section{Reference}

(1) Sluiter, A. Determination of Structural Carbohydrates and Lignin in Biomass: Laboratory Analytical Procedure (LAP); Issue Date: 7/17/2005. Tech. Rep. 2008, 16.

(2) Deye, J. F.; Berger, T. A.; Anderson, A. G. Nile Red as a Solvatochromic Dye for Measuring Solvent Strength in Normal Liquids and Mixtures of Normal Liquids with Supercritical and near Critical Fluids. Anal. Chem. 1990, 62 (6), 615-622. 10.1021/ac00205a015.

(3) Jessop, P. G.; Jessop, D. A.; Fu, D.; Phan, L. Solvatochromic Parameters for Solvents of Interest in Green Chemistry. Green Chem. 2012, 14 (5), 1245. 10.1039/c2gc16670d.

(4) Kamlet, M. J.; Taft, R. W. The Solvatochromic Comparison Method. I. The .Beta.-Scale of Solvent Hydrogen-Bond Acceptor (HBA) Basicities. J. Am. Chem. Soc. 1976, 98 (2), 377-383. 10.1021/ja00418a009.

(5) Mellein, B. R.; Aki, S. N. V. K.; Ladewski, R. L.; Brennecke, J. F. Solvatochromic Studies of Ionic Liquid/Organic Mixtures. J. Phys. Chem. B 2007, 111 (1), 131-138. 10.1021/jp0653353.

(6) Taft, R. W.; Kamlet, M. J. The Solvatochromic Comparison Method. 2. The .Alpha.-Scale of Solvent Hydrogen-Bond Donor (HBD) Acidities. J. Am. Chem. Soc. 1976, 98 (10), 2886-2894. 10.1021/ja00426a036.

(7) Xia, Q.; Liu, Y.; Meng, J.; Cheng, W.; Chen, W.; Liu, S.; Liu, Y.; Li, J.; Yu, H. Multiple Hydrogen Bond Coordination in Three-Constituent Deep Eutectic Solvents Enhances Lignin Fractionation from Biomass. Green Chem. 2018, 20 (12), 2711-2721. 10.1039/C8GC00900G. 